\title{
IJIE
}

\section{Construction of Driving Seat Frame and 2-Dof Motion Simulator}

\author{
I.M. Saadon ${ }^{*}$, N. Idris ${ }^{2}$, E.Z. Ahmad ${ }^{1}$, H.M. Yassim¹, N. Norddin ${ }^{1}$ \\ ${ }^{1}$ Faculty of Electrical \& Engineering Technology, \\ Universiti Teknikal Malaysia Melaka, 75450 Melaka, MALAYSIA \\ ${ }^{2}$ Faculty of Mechanical \& Manufacturing Technology, \\ Universiti Teknikal Malaysia Melaka, 75450 Melaka, MALAYSIA \\ *Corresponding Author
}

DOI: https://doi.org/10.30880/ijie.2020.12.05.019

Received 29 January 2020; Accepted 4 March 2020; Available online 30 June 2020

\begin{abstract}
Driving simulators are increasingly being used as a didactic tool in car racing games because it offers virtual driving experience to users. However, existing driving simulators are found in market at high cost. This paper proposes the design and development of a low cost 2-DOF driving motion simulator with the interface from SimTools. The driving simulator comprises a steering wheel, a pedal and simulator software. When the steering wheel is turned in the racing simulator Live for Speed, it controls the angle of the driver's seat upraising. The input data from the steering wheel is derived using SimTools, which extracts the motion data from the racing simulator. Potentiometer is used as the feedback sensor that manipulates the data from SimTools to control the angle of the dc motor driver rotation. The motion is created using the rotation of two dc motors that actuate the car seat frame. Moto Monster motor driver which supports 30A of current is used to control both of the dc motors using Arduino microcontroller. The angle control of the driver's seat is proportional to the input data from steering wheel. This paper contributes the basic idea on building a low cost motion simulator.
\end{abstract}

Keywords: Defender Steering Wheel; Driving Simulator; Moto Monster Motor Driver; SimTools

\section{Introduction}

The effectiveness of driving motion simulators is related to their capabilities of well reproducing the driving sensations, and hence, it is crucial that the motion control strategies generate both realistic and feasible inputs to the platform, to ensure that it is kept within limited operation space. Therefore, it can be used in many different areas such as for research purposes to study the behaviour of the driver or develop and evaluate the new subsystems of the vehicle, or they can be used for training of drivers or for entertainment as in video games [1]. The creation of simulators makes learning more convenient and cheaper, and less dangerous, especially when training actions in various emergency situations [2]. Industrial simulation is significant to improve the understanding of a process. Engineers perform different simulations to maximize trialing of the product, predict flaws, improve ergonomic aspects, and reduce operational cost for experimentation. It also enables them for multiple recreation of the design before it is made available and construct new and robust plans. Thru simulation, topographical alterations become easier and appropriateas manufactured models came from different models of simulations [3]. Advantages of using a driving simulator are safety (there are no traffic accidents during driving) and simple collecting of data related to the driver's behavior [4].

When picking motion, there are more than 6 degree of freedom (DOF) can be configurable as needed. The 6 most common motion is roll, pitch, yaw, surge, sway and heave. Many motion simulators with vary degree of freedom are invented to achieved the capabilities of well reproducing the driving sensations [5]. For this paper, a 2-DOF motion derived from roll and pitch are developed as the basic motion for a car. 
On the game side, the telemetry and motion data extractor program uses the InSim.Net open source library to connect to the game or simulator and share packets of data. This library was developed by the racing simulator Live for Speed. For this paper is the library is relevant in providing the methods and events for extracting motion data from the simulator [6]. Fig. 1 denotes the flow chart of the operation of the driving motion simulator. The process begins with the initial position where the potentiometer value equals to SimTools hexa value. When steering turns left or right, and pedal accelerates or brakes, SimTools hexa value will change accordingly, hence it moves the dc motor until the potentiometer value is equal to SimTools hexa value. The motor continues to rotate forward or reverse until the initial value is achieved.

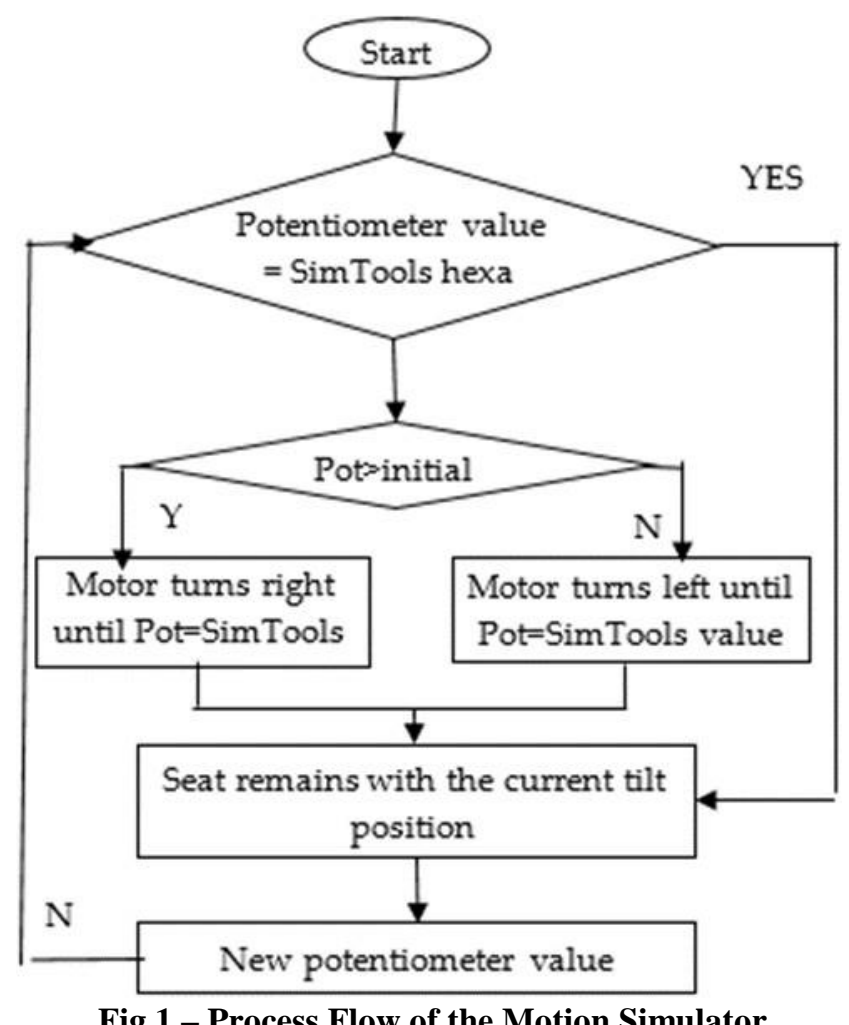

Simtools software transmits the hexadecimal data value to Arduino microcontroller which converts the hexadecimal number to the decimal number. The decimal number is then sent to update the potentiometer value. The motor starts turning based on the updated value of potentiometer. For this whole looping, the variable value from Simtools lets the potentiometer analog value to vary accordingly causing the dc motor to rotate hence the seat frame that is coupled to the de motor moves according to the rotation.

\section{Methodology}

\subsection{Driver's Seat Frame Design and Setup}

Fig. 2 shows the dimension of driving motion simulator frame seat in real model. Based on this figure, the total area of the base is $(42 \times 110) \mathrm{cm} 2$. The width of the bucket seat is $49 \mathrm{~cm}$. A moveable seat within certain axis is required so when the user input the data to microcontroller, the two of the motor angle will change and caused the seat to move along with the angle of the dc motor. Fig. 3 shows the complete frame seat. The steel plate is used in this project to ensure a good stability and endurance. For the gimbal movement of the seat, car gimbal U-joint is used. The bottom of the car U-joint are welded to a steel plate so that it is strong enough to support the load. Since the load of the seat is below $20 \mathrm{~kg}$ without a person, a spring from motorcycle absorber is added to support the U-joint and the dc motor actuator. The spring helps to reduce the load on the top by retracting, attracting and bending itself downward so that the angle prompt is achieved. The dc motor shaft is connected with the car steering rod since the end of the steering road is moveable which will give a smooth transition of the dc motor movement toward the seat frame.

\subsection{Driving Motion Simulator}

Fig. 4 represents the electrical circuit in this paper for the development of a driving motion simulator. Each potentiometer will be coupled to the dc motor shaft. Power supply unit is connected to the Moto Monster dc motor 
driver because the dc motor rating power is 320 watt in total. SimTools will be used in this paper to extract the motion data of the simulator car from the simulator. The extracted data will be send to the Arduino to be processed into an analog potentiometer value. This potentiometer will then determine the rotation and position of the dc motor.

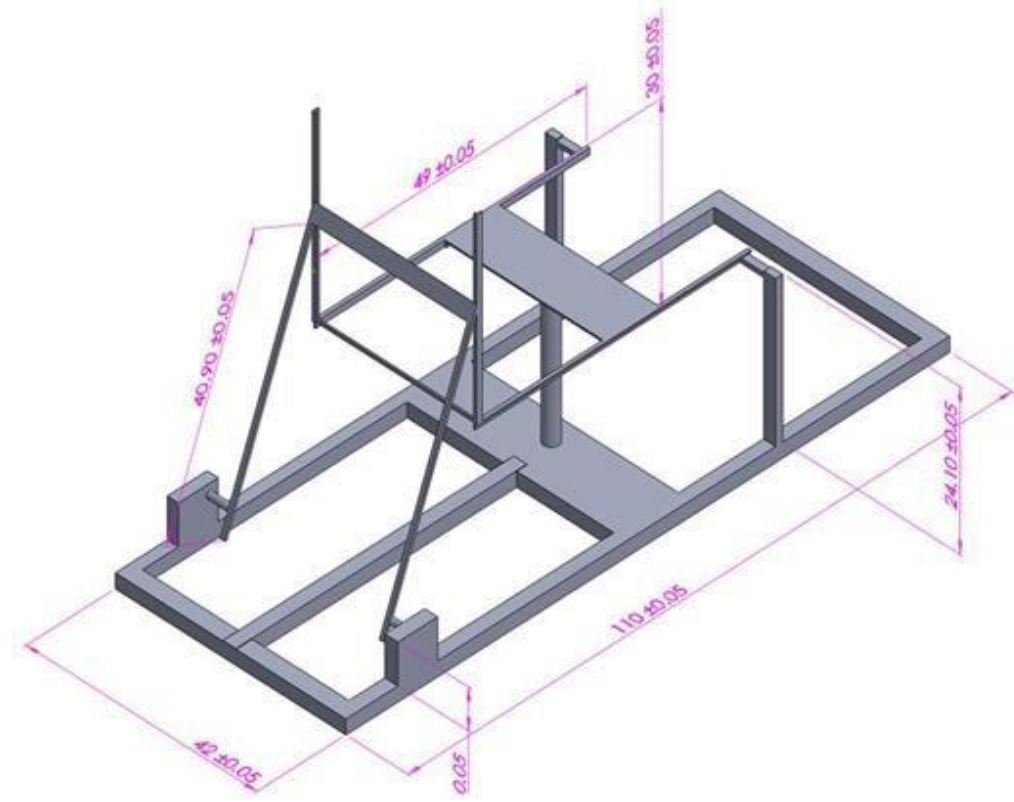

Fig.2 - Motion simulator frame seat design

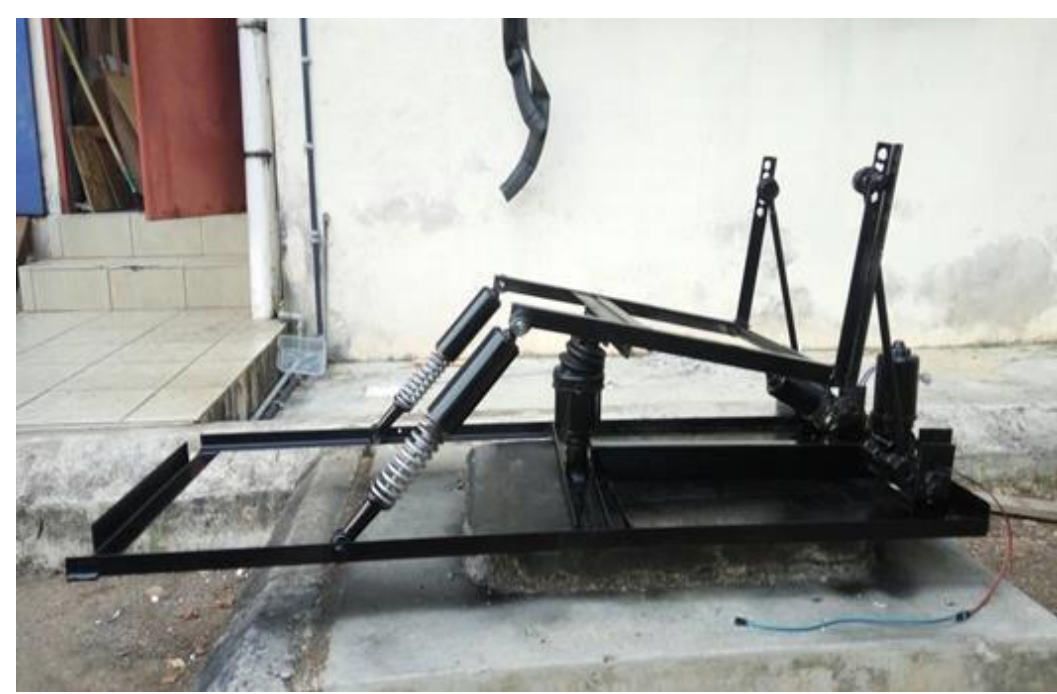

Fig. 3 - Motion simulator frame seat

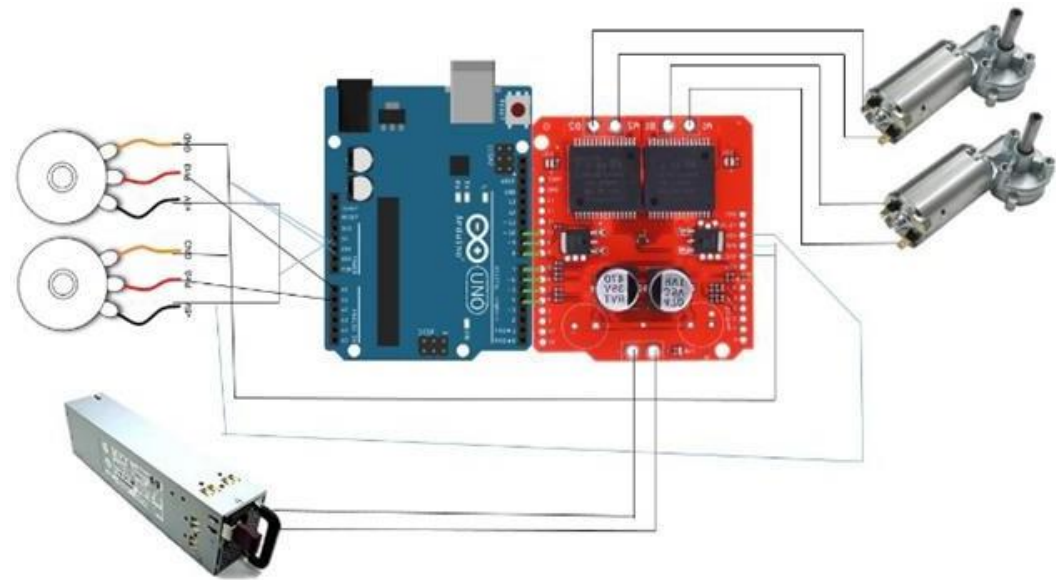

Fig. 4 - Block Diagram of the Motion Simulator 
The selection of the DC motor is chosen because of the cost. The excellent features of DC motor of high mechanical strength, durability, reliability, effectiveness, portability, speed and cheap cost of maintenance, have placed them ahead as a preferable horsepower control motor [7]. A powerful servo motor cost a large amount of money while the DC motor is a lot cheaper. The combination of potentiometer and DC motor will produce a servo motor. The type of dc motor used in this paper is used $12 \mathrm{~V}$ car wiper motor. Wiper motor is a direct current motor that installed almost in any car. The motor is used to move the attached wiper by a high torque rotation. The voltage rating for the dc motor is $12 \mathrm{~V}$. The maximum current rating with full load is $16 \mathrm{~A}$. The power consumption is about $80 \mathrm{~W}$. At no-load speed, the motor can turn $45 \mathrm{rpm}$ while in working speed $38 \mathrm{rpm}$.

In order to drive the $12 \mathrm{~V}$ wiper dc motor, the needed of DC motor driver is compulsory. The DC motor driver can control the speed as well as the direction of rotation of the motor. Monster Motor Shield VNH2SP30 is the motor driver used in this simulator. The motor driver can control 2 DC motor simultaneously. It is a full bridge motor driver intended for wide range of automotive applications that allows the control of relatively powerful DC motors. Generally, it consists of four MOSFETs forming an H-Bridge. For DC motor, the H-Bridge driver can be used to control the direction and speed of DC motor. Therefore, it has become an important driving component for motor control [8]. However, both the function can be performed using another type of motor driver consisting only one MOSFET and two relays [9].

Finally, the DC motor will act as actuator for the seat thus provide a motion for the seat frame. This process will continuously run as long as the SimTools software provide the motion data. The main component to control the angle of the dc motor is potentiometer that acts as a feedback loop for the rotation of the motor on when to rotate left, rotate right and stop. A potentiometer is a three-terminal resistor with sliding or rotating contact that forms an adjustable voltage divider. If only 2 terminals are used, one end and the wiper, it acts as a variable resistor or rheostat. Potentiometer will be used as a feedback for the position of the motor. The variation of seat tilt (from $5 \circ$ to $-5^{\circ}$ and vice versa) performed periodically with low speed mitigates the discomfort perception [10].

If the potentiometer is entirely twisted to max which is 1024 , the Arduino interprets the potentiometer data value as motor position, Arduino considers that the motor is now in maximum position, but the instruction is to be in middle position of potentiometer. Therefore, the Arduino will request the motor to go opposite in order to go to the middle position. While the potentiometer value is not 512 (middle), the motor will keep turning. In the code, the tolerance parameter is added so as the potentiometer is around the middle position (<mid+tolerance and >mid+tolerance) the motor stops.

\section{Results}

For the car simulator, Live For Speed program is used in this paper. Fig.5 shows the Live for Speed software link with SimTools Virtual axis. The virtual axis shows the motion involved when driving a car simulation. Since only Roll and Pitch is available in the motion seat driving motion simulation, the other motion can be used for future development of motion seat.

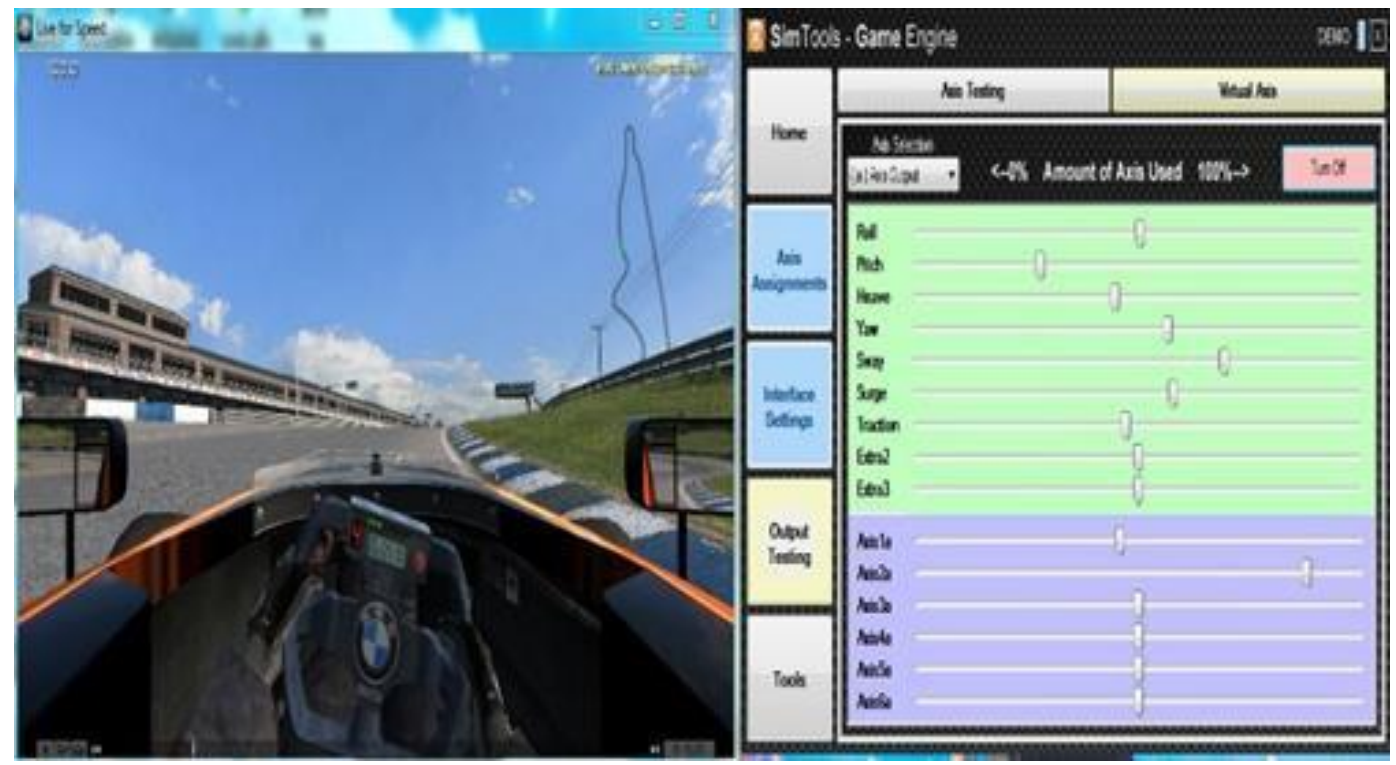

Fig. 5 - Live for Speed Simulator

The SimTools software is then connected with the arduino and motor driver to test the rotation of the dc motor and the motion of the seat. Fig.6(a), (b), (c), (d) and (e) show the motion of the seat when the SimTools Axis testing is vary at initial, $-80 \%$ roll, $+80 \%$ roll, $-80 \%$ pitch and $+80 \%$ pitch. The motion seat driving simulation is tested with load person which is below 60 kilogram. As shown in the figures, at initial position, the seat is at $0^{\circ}$ movement. When the 
steering is turned to the right, the maximum roll is set to $80 \%$ and this causes the frame seat to tilt to $5 \%$ from the right. Meanwhile when the steering is turned to the left, the maximum roll is set to $-80 \%$, causing the seat to tilt $5 \%$ to the right. Maximum speeding of $80 \%$ pitch causes the seat to tilt $5 \%$ to the back and maximum braking of $80 \%$ causes the seat to tilt $5 \%$ to the front.

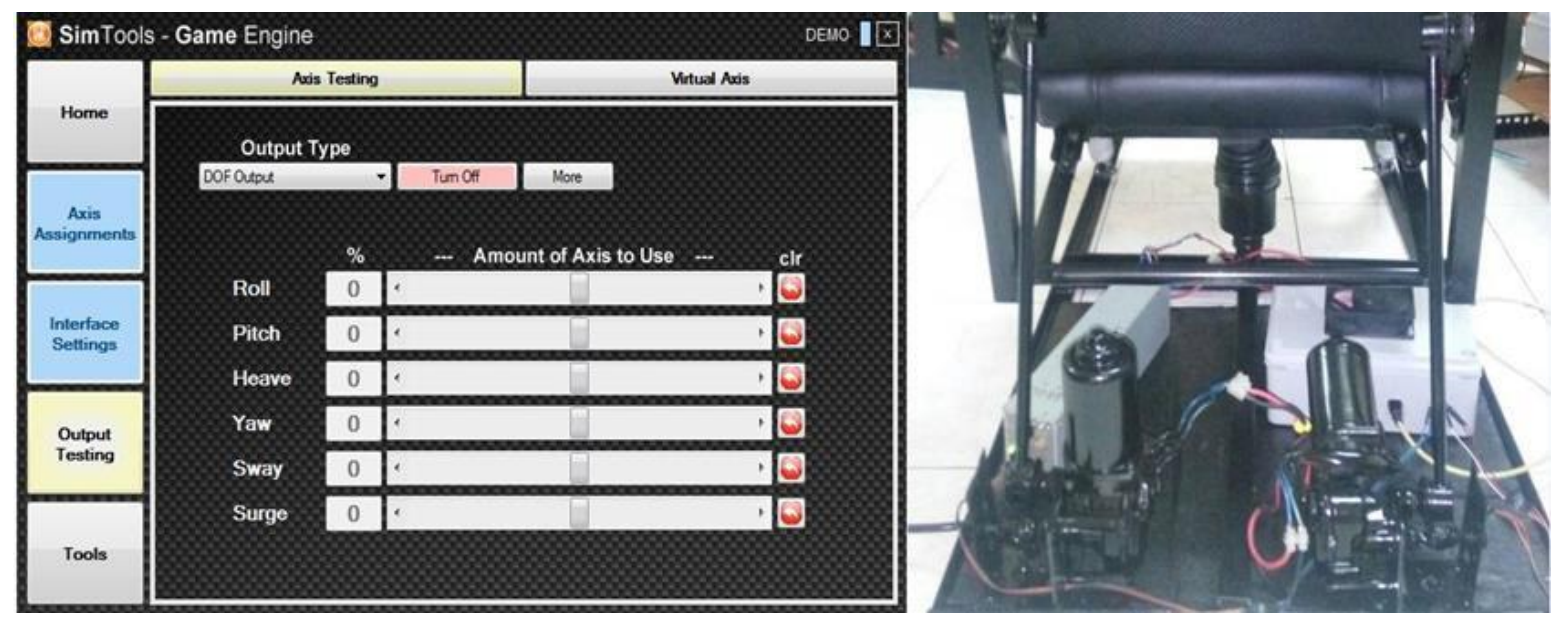

Fig. 6 (a) - The Seat Motion at Initial Position
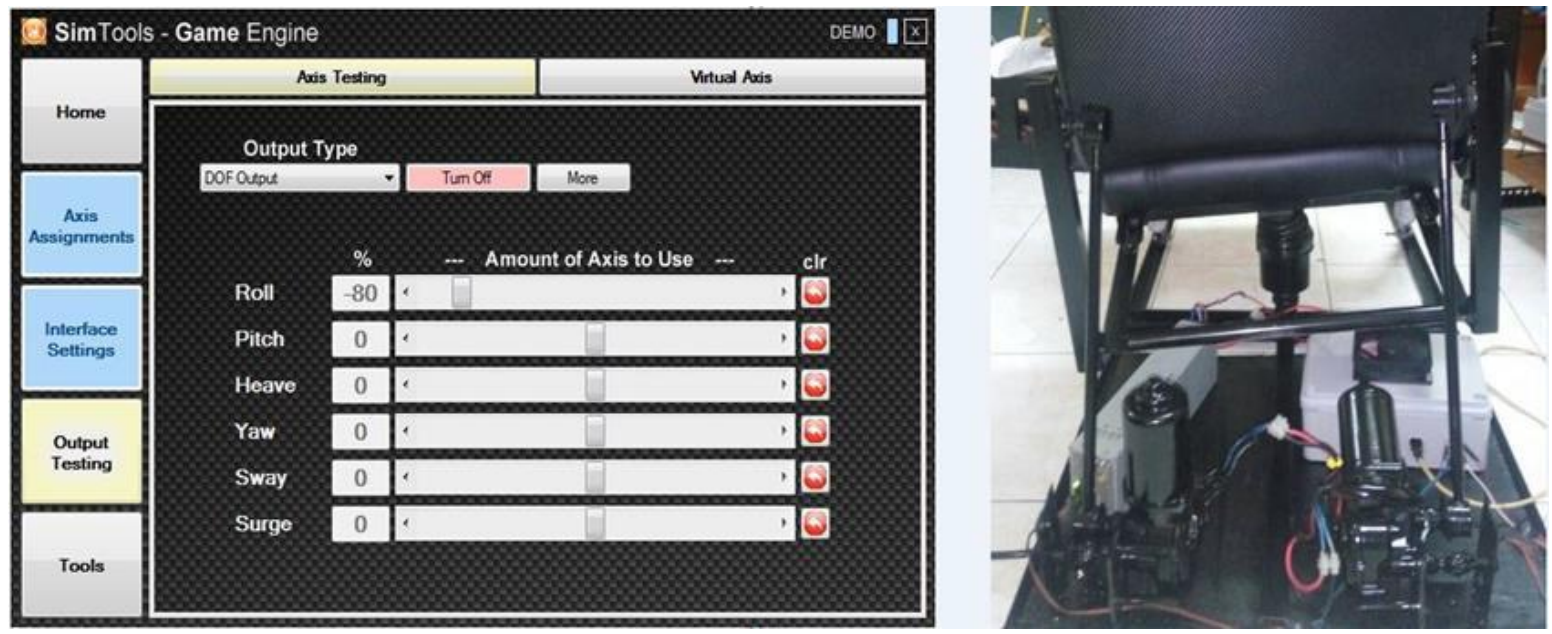

Fig. 6 (b) - The Seat Motion at $-80 \%$ Roll

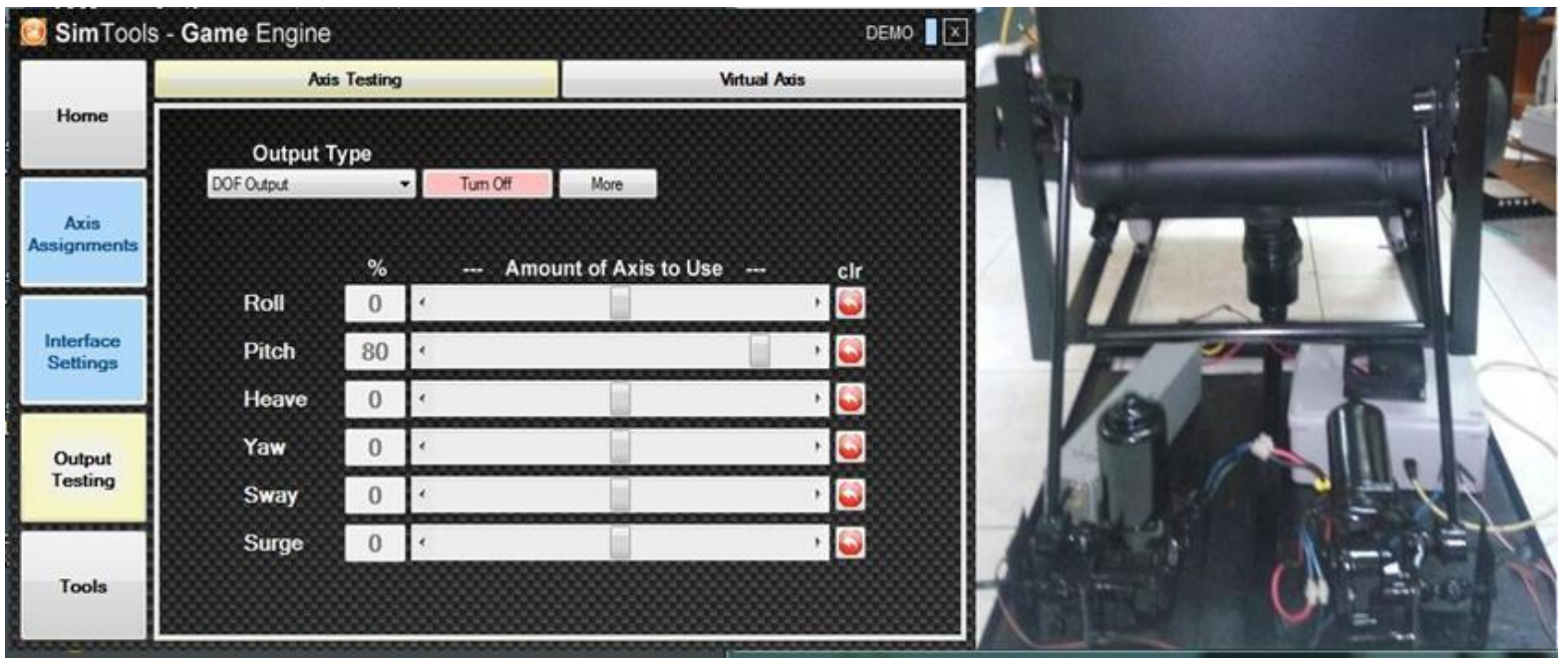

Fig. 6 (c) - The Seat Motion at $+80 \%$ Roll 


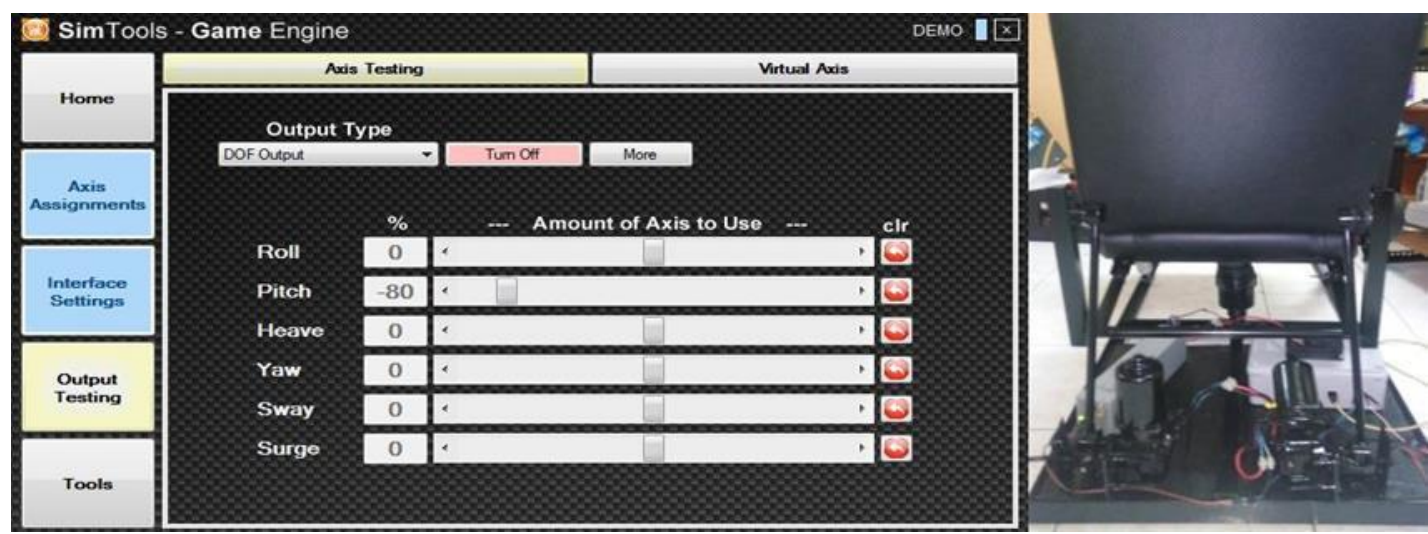

Fig. 6 (d) - The Seat Motion at -80\% Pitch

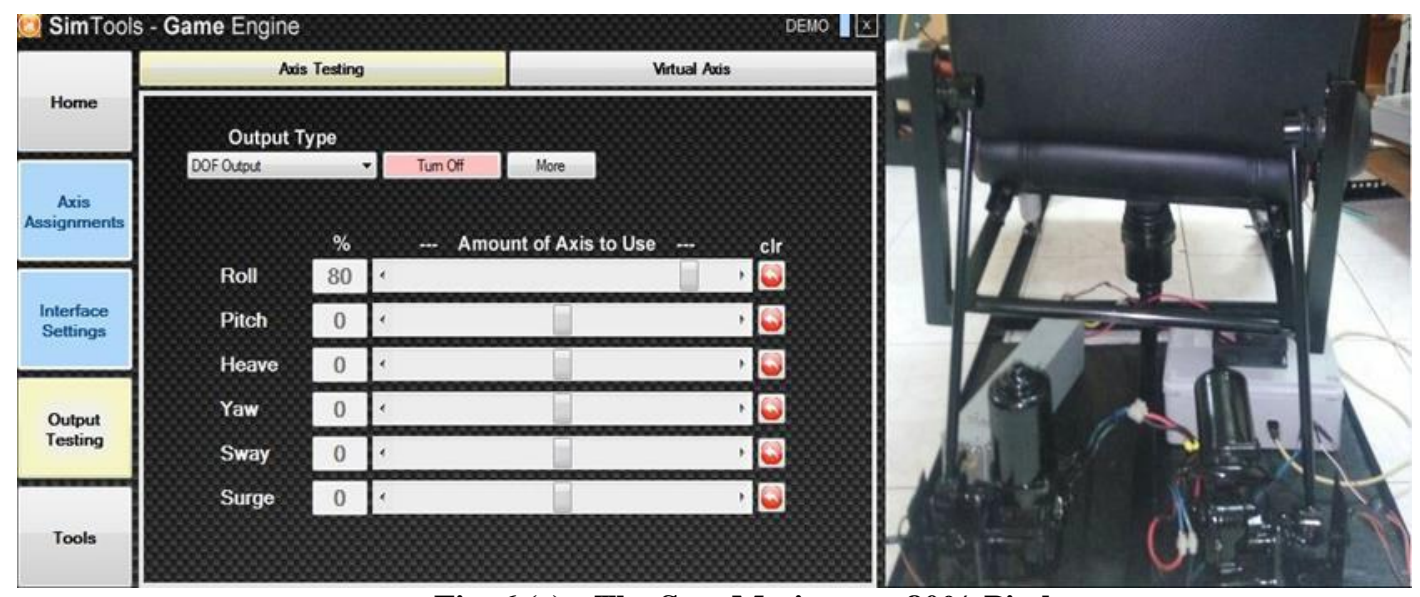

Fig. 6 (e) - The Seat Motion at $+80 \%$ Pitch

\section{Conclusion}

The motion seat for driving car simulator is developed for controlling the angle of dc motor which actuates the designed frame seat by using data extracted from the SimTools software to give more realistic behaviour and feels for the experience to be a driver when driving car simulator instead of a static driving simulation. This will drastically increase the excitement of user entertainment and promote higher level of simulation environment at once increase their awareness behaviour. This paper provides a 2 degree of freedom motion of a car, since there are 6 degree of freedom motion for a car, it is recommended to increase the degree of freedom of this paper. The paper experience can be maximized by upgrading the platform so that it can support the static monitor and controller. Other than that, to eliminate the limitation of weight the motor can support, a higher torque and power rating is suggested to ensure that the load limitation can be maximize.

\section{Acknowledgment}

This work was supported by the Universiti Teknikal Malaysia Melaka (UTeM), Ministry of Education (MOE) are greatly acknowledged. The research was funded by Research Acculturation Grant Scheme Grant No. (RAGS/1/2015/TK0/UTEM/03/B00122).

\section{References}

[1] Slob, J.J., (2008) State-of-the-Art Driving Simulators, a Literature Survey, DCT report, Eindhoven University of Technology.

[2] Evgeny I. Krasnov, (2015). Development of a Motion System for a Training Simulator Based on Stewart Platform. 1-2.

[3] Wilfred Ralph G. Gomez (2019), Design and Development of an Integrated Automation Simulation System, The International Journal of Integrated Engineering (IJIE), Vol.11 No.8, 41-54.

[4] L. Banjanovic-Mehmedovic, R. Stojak and S. Kasapovic, (2016) Driving behavior simulator of lane changing using user-designed interface, 2016 5th Mediterranean Conference on Embedded Computing (MECO), Bar, 493-497. 
[5] Mattia Bruschetta, Fabio Maran, and Alessandro Beghi, (2016). A Nonlinear, MPC-Based Motion Cueing Algorithm for a High-Performance, Nine-DOF Dynamic Simulator Platform. IEEE transactions on Control Systems Technology. 1-2.

[6] N. M. Sultan, Badrul Aisham Md Zain, Fatin Farhana Anuar, M. S. Yahya, Idris Abdul Latif, M. K. Hat,Sami Al-alimi (2019), Modeling and Speed Control for Sensorless DC Motor BLDC Based on Real Time Experiment, International Journal Of Integrated Engineering (IJIE), Vol.11 no.8, 55-64.

[7] X-Sim Extractor Motion Driver Guide (2012)

[8] Hsin-Chuan Chen, (2013). An H-Bridge Driver Using Gate Bias for DC Motor Control. IEEE 17th International Symposium on Consumer Electronics (ISCE).

[9] G. Mulay, A. Yembarwar and S. Raje, (2014) A DC motor driver consisting of a single MOSFET with capability of speed and direction control," 2014 IEEE 6th India International Conference on Power Electronics (IICPE), Kurukshetra, 1-3.

[10] Fernanda Maradeia, Leonardo Quintanab and Javier Castellanosc (2016) Influence of Seat Tilt Motion on Discomfort Perception During a Simulated Driving Activity, US National Library of Medicine. 